\title{
The Value of Endometrial Volume as Estimated by Three-Dimensional Ultrasound for Detecting Endometrial Cancer in Postmenopausal Women: A Systematic Review and Meta-Analysis
}

\author{
Authors: \\ Cristian Morales, ${ }^{1}$ Raul Diago, ${ }^{2}$ Salvador Cortés, ${ }^{3}$ Carla Peixoto, ${ }^{4}$ Maria \\ Ángela Pascual, ${ }^{5}$ Stefano Guerriero, ${ }^{6}{ }^{*}$ Juan Luis Alcázar ${ }^{7}$ \\ 1. Department of Obstetrics and Gynecology, Hospital San Juan de Dios, Santiago, \\ Chile \\ 2. Department of Obstetrics and Gynecology, Hospital Universitario Severo Ochoa, \\ Madrid, Spain \\ 3. Department of Obstetrics and Gynecology, Hospital Universitario Virgen de la \\ Arrixaca, Murcia, Spain \\ 4. Department of Obstetrics and Gynecology, Centro Hospitalar São João, Porto, \\ Portugal \\ 5. Department of Obstetrics, Gynecology, and Reproduction, Hospital Universitario \\ Dexeus, Barcelona, Spain \\ 6. Department of Obstetrics and Gynecology, Policlinico Universitario Duilio Casula, \\ Cagliari, Italy \\ 7. Department of Obstetrics and Gynecology, Clinica Universidad de Navarra, \\ Pamplona, Spain \\ *Correspondence to jlalcazar@unav.es
}

Disclosure: $\quad$ The authors have declared no conflicts of interest.

Received:

11.03 .20

Accepted:

14.04 .20

Keywords:

Cancer, endometrial, endometrial volume, three-dimensional (3D) ultrasound.

Citation:

EMJ Radiol. 2020;1[1]:79-88.

\section{Abstract}

Objective: To analyse the diagnostic performance of endometrial volume calculated by three-dimensional (3D) ultrasound for diagnosing endometrial carcinoma in women with postmenopausal bleeding.

Methods: An extensive search of papers analysing the role of endometrial volume calculated by 3D ultrasound for diagnosing endometrial carcinoma in women with postmenopausal bleeding was performed in MEDLINE/PubMed and Web of Science from January 1996 to January 2020. Quality was assessed using the Quality Assessment of Diagnostic Accuracy Studies 2 (QUADAS-2) tool.

Results: The extended search identified 318 citations but after exclusions, eight articles were included in the meta-analysis. The risk of bias for most studies was high for the four domains assessed in QUADAS-2. Overall, after excluding three studies that contributed significantly to heterogeneity, pooled estimated sensitivity and specificity for diagnosing endometrial cancer were $87 \%$ (95\% confidence interval: 80-92\%) and 60\% (95\% confidence interval: 51-68\%), respectively. Heterogeneity was low or moderate. 
Conclusion: Endometrial volume as estimated by 3D ultrasound using virtual organ computer-aided analysis (VOCAL ${ }^{\mathrm{TM}}$ ) software has a moderate diagnostic performance for detecting endometrial malignancy in women with postmenopausal bleeding.

\section{INTRODUCTION}

\section{METHODS}

Endometrial carcinoma is the most frequent gynaecological malignancy in western countries, with most of the patients being postmenopausal. ' The main symptom of this disease is postmenopausal bleeding. The first approach to take in a woman who is symptomatic is to evaluate the endometrial thickness using two-dimensional ultrasound because a endometrial thickness $<5 \mathrm{~mm}$ has a very high negative-predictive value (99.3\%) when ruling out endometrial cancer, meaning that unnecessary biopsies can be avoided. ${ }^{2}$ In contrast, a thickened endometrium $(>5 \mathrm{~mm})$ is a relatively nonspecific finding that can be found in many benign endometrial pathologies, such as cyst atrophy, polyp, or non-atypical hyperplasia. In fact, the specificity reported is approximately $50.0 \%$. $^{2,3}$

In the last two decades, three-dimensional (3D) ultrasound has become available for the diagnosis of some gynaecological diseases. Currently, 3D ultrasound is considered the firstline imaging diagnostic technique for some gynaecological lesions, such as congenital uterine anomalies. ${ }^{4}$ Furthermore, extensive research using this technique has been reported in the fields of reproductive medicine $^{5}$ and gynaecological oncology. ${ }^{6}$

The estimation of endometrial volume using 3D ultrasound is accurate ${ }^{7}$ and reproducible among examiners. ${ }^{8,9}$ Specifically, the role of the endometrial volume for diagnosing endometrial carcinoma in women with postmenopausal bleeding has been evaluated in a small number of small-scale prospective studies since the first report on its use in $1996 . .^{10}$ However, the role of this technique as a diagnostic test in this clinical setting has not been clearly established.

The aim of this systematic review and metaanalysis is to evaluate the diagnostic performance of the endometrial volume calculated by 3D ultrasound for diagnosing endometrial carcinoma in symptomatic postmenopausal women.

\section{Protocol and Registration}

This meta-analysis has been performed according to the PRISMA statement and the Synthesizing Evidence from Diagnostic Accuracy Tests (SEDATE) guidelines." The protocol was not registered, a decision made by the researchers to avoid delays in starting the meta-analysis. All inclusion and exclusion criteria for studies to be selected were defined, as well as how data extraction and quality assessment had to be performed before starting the data search. Because of the study's nature and design, Institutional Review Board (IRB) approval was waived.

\section{Data Sources and Searches}

Two of the authors (SC and CM) screened two electronic databases, MEDLINE/PubMed and Web of Science, to identify potentially eligible studies published from January 1996 to January 2020. The search terms included and captured the concepts of "endometrial cancer", "endometrial malignancy", "three-dimensional ultrasound", "postmenopausal bleeding", and "endometrial volume". The language limit was set to English.

\section{Study Selection and Data Collection}

Two authors (CM and RD) screened the titles and abstracts identified by the search to exclude irrelevant articles. Then, full-text articles were selected to identify potentially eligible studies by applying set criteria:

$>$ prospective and retrospective cohort studies that included patients with postmenopausal bleeding who underwent transvaginal ultrasonography examinations and included the calculation of endometrial volume using the virtual organ computer-aided analysis (VOCAL ${ }^{\mathrm{TM}}$ ) method;

$>$ histological findings evaluated with endometrial samplings or hysterectomy;

$>$ presence of data reported that would allow construction of a $2 \times 2$ table with a specific 
cut-off of endometrial volume to estimate the diagnostic accuracy.

To avoid inclusion of duplicate cohorts from at least two studies reported from the same authors, the study period of each study was examined; if dates overlapped, the latest study published was selected. Additional articles were searched by reading the reference lists of those articles selected for full-text reading. The patient, intervention, comparator, outcome, and study design criteria used for inclusion and exclusion of studies were recorded.

The authors had intention to assess data based on individual patient information; therefore, they contacted the authors from the selected studies asking for specific data about some clinical characteristics of the patients, 3D ultrasound endometrial volume estimation results, and histologic data. This way, using the predefined endometrial volume, thresholds reported from the authors in the respective paper could be avoided. However, no responses were received from any of the authors. Therefore, the quantitative analysis using the respective threshold reported in each paper was performed.

Diagnostic accuracy results from the selected studies were retrieved independently by two authors (CM and RD). Disagreements arising during the process of study selection and data extraction were resolved by consensus among all four authors.

\section{Risk of Bias in Individual Studies}

A quality assessment of studies included in the meta-analysis was conducted by using the tool provided by the Quality Assessment of Diagnostic Accuracy Studies 2 (QUADAS-2).12 The QUADAS-2 format includes four domains: 1) patient selection; 2) index test; 3) reference standard; and 4) flow and timing. For each domain, the risk of bias and concerns about applicability (the latter not applying to the domain of flow and timing) were analysed and rated as low, high, or unclear risk. The quality assessment was used to provide an evaluation of the overall quality of the studies and to investigate potential sources of heterogeneity.

Three authors (CM, RD, and JLA) evaluated the methodological quality independently. Disagreements were solved by discussion between these authors. The assessment of the quality was based on several issues, depending on the domain assessed. For the patient selection domain, the authors considered whether the study described the study's design (in retrospective studies in which the reference test was already known by the researchers when performing the index test could not be elucidated, the worst case scenario was opted for and these were considered studies with a high risk of bias) as well as patients' inclusion and exclusion criteria. For the index test domain, whether the study reported on the method of 3D volume acquisition and how the volume was calculated was considered, as well as how this was performed and interpreted. For the reference test domain, whether the study reported on the reference standard used (histology or not) and how sample was obtained was considered. Finally, for the flow-and-timing domain, the authors considered whether the study reported on the time elapsed from the index test assessment to the reference test (more than 4 weeks from index test to reference test was considered as high risk for bias).

\section{Statistical Analysis}

Information on the diagnostic performance of endometrial volume was extracted. A bivariate model was used to estimate the pooled sensitivity, specificity, positive likelihood ratio (LR), and negative LR. The LR were used to characterise the clinical utility of a test and to estimate the post-test probability of disease..$^{13}$ Using $8 \%$ prevalence of endometrial cancer in women with postmenopausal bleeding (pretest probability), ${ }^{2}$ post-test probabilities were calculated by the positive and negative LR and plotted on a Fagan nomogram.

Heterogeneity for sensitivity and specificity was assessed by the Cochran's $Q$ statistic and the heterogeneity $\mathrm{I}^{2}$ index. $^{14}$ A $\mathrm{p}$ value $<0.1$ indicated heterogeneity, and $\mathrm{I}^{2}$ values of $<25 \%$, $25-50 \%$, and $>75 \%$ were considered to indicate low, moderate, and high heterogeneity, respectively.

Forest plots of sensitivity and specificity of all studies were plotted. Considering that it could be a threshold effect, given that different studies used different thresholds for endometrial volume, a bivariate random effects modelling of 
sensitivity and specificity was used to identify how much the threshold effect could explain heterogeneity, if found.

Hierarchical summary receiver operating characteristic curves were plotted to illustrate the relationship between sensitivity and specificity. Additionally, a binomial exact distribution for assessing within-study variability for sensitivity and specificity was used. Publication bias was assessed by the method of Deeks et al. ${ }^{15}$

All analyses were performed with MIDAS and METANDI commands in Stata version 12.0 software for Windows (StataCorp, College Station, Texas, USA). A $\mathrm{p}$ value $<0.05$ was considered statistically significant.

\section{RESULTS}

\section{Search Results}

The electronic search provided 318 citations. After exclusion of 120 duplicate records, 198 citations remained. Of these, 166 were excluded because it was clear from the title or abstract that they were not relevant to the review (studies not related to the topic [ $n=146]$, reviews [ $n=9]$, articles published in non-English languages $[n=8]$, and letters to Editor $[n=3])$.

The full text of the remaining articles was read. A further 24 studies were excluded: two studies did not use the VOCAL method; 14 studies included only patients with previous diagnosis of carcinoma; four studies included premenopausal and postmenopausal women and data could not be stratified for menopausal status; in three studies it was not possible to retrieve data to make a $2 \times 2$ table to calculate true positive, true negative, false positive, and false negative cases; and one study was a retrospective study using the same data of another included study. The remaining eight ${ }^{16-23}$ studies were ultimately included in the present meta-analysis. No additional studies from references cited in these eight studies were found.

\section{Characteristics of the Included Studies}

Eight studies published between 2007 and 2013 reporting on 981 patients were included in the final analyses. Among these 981 women, 267 had a malignant lesion. The mean prevalence of malignant lesions was $27.2 \%$, ranging from $10.4 \%$ to $47.0 \%$. All studies reported some clinical characteristics of the patients. All patients were women with postmenopausal bleeding. Postmenopausal was defined as, at least, 1 year of amenorrhoea in all studies. Pathologic confirmation obtained after endometrial biopsy was reported in all studies.

\section{Methodological Quality of the Included Studies}

The study design was clearly stated as prospective in all the studies. The QUADAS-2 assessment of the risk of bias and concerns regarding applicability of the selected studies is shown in Figure 1.

With regard to the risk of bias for the patient selection domain, all studies were considered as having a high risk of bias. Six out of the eight studies included only women with a thickened endometrium, >4 $\mathrm{mm} ; 7^{17-20,22,23}$ three studies excluded patients with previous gynaecologic disease such as fibroids or polyps:16,21,23 and one study pooled the hyperplasia with atypia and endometrial cancer in the same group. ${ }^{16}$ Concerning the index test domain, all the studies used the VOCAL rotational method to calculate the endometrial volume. In seven studies, the method of the index text as well as how it was performed was adequately described. One study did not describe the angle rotation step used. ${ }^{22}$ However, five studies ${ }^{16,17,19,20,23}$ were considered at high risk because they used a $30^{\circ}$ rotation step for endometrial volume acquisition, and it has been shown that this approach is less reliable than using $9^{\circ}$ or $15^{\circ} .{ }^{24,25}$ Only two studies used less than $30^{\circ}$ rotation step, and they were considered as having low risk for bias regarding the index test. ${ }^{18,21}$

For the reference standard domain, all studies were considered low risk because all patients were studied with endometrial sampling and posterior histologic diagnosis. Regarding the flow and timing domain, in four studies the time elapsed between the index test and reference standard was less than 1 week, ${ }^{17,18,20,23}$ but in four studies it was unclear. 16,19,21,22

Concerning applicability, for the patient selection domain, index test, and referent test, all studies were considered low risk for applicability because they used an adequate technique (transvaginal ultrasound) in the adequate clinical setting (postmenopausal bleeding) with an adequate reference standard (endometrial biopsy). 

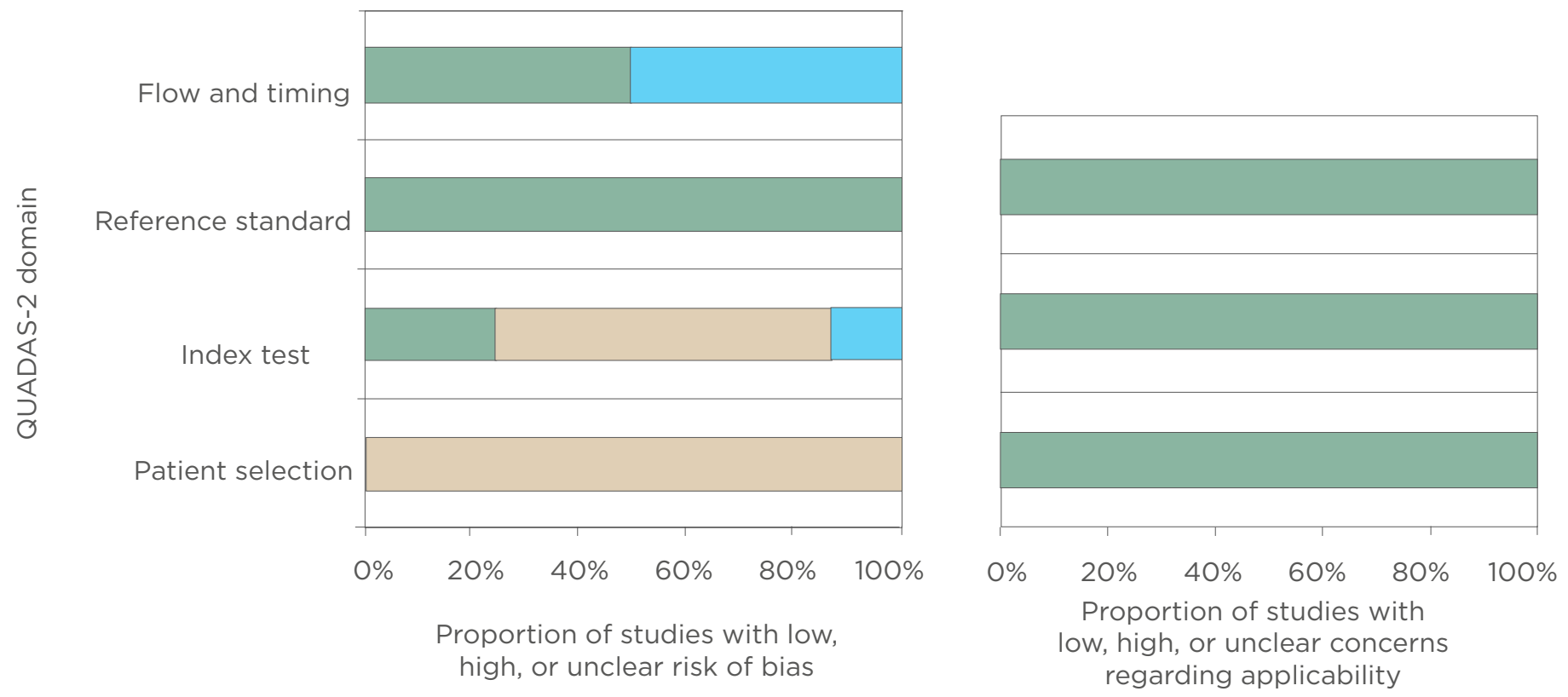

$\square$ Low $\square$ High $\square$ Unclear

Figure 1: Histogram plot showing quality assessment (risk of bias and concerns about applicability) for all studies included in the meta-analysis.

QUADAS-2: Quality Assessment of Diagnostic Accuracy Studies 2.

\section{Diagnostic Performance of Endometrial Volume for Detection of Endometrial Cancer}

The pooled sensitivity, specificity, positive LR, and negative LR of endometrial volume for detecting endometrial cancer were $87 \%$ (95\% confidence interval [Cl]: 77-93\%), 69\% (95\% Cl: 54-82\%), 2.8 (95\% Cl: 1.9-4.2), and 0.19 (95\% Cl: 0.12-0.30), respectively. The diagnostic odds ratio was 15.0 (95\% Cl: 9.0-24.0). Significant heterogeneity was found for sensitivity ( $\left.\right|^{2} \quad=74.48 \%$; Cochran $Q$ $=27.43 ; \quad p<0.001)$ and specificity $\left(1^{2}=93.45 \%\right.$; Cochran $Q=106.89 ; p<0.001)$. Bivariate modelling showed that a threshold effect explained this heterogeneity with three studies involved. $16,17,19$

After excluding these three studies, pooled sensitivity, specificity, positive LR, and negative LR of endometrial volume for detecting endometrial cancer were 87\% (95\% Cl: 80-92\%), 60\% (95\% Cl: 51-68\%), 2.2 (95\% Cl: 1.7-2.7), and 0.22 (95\% Cl: 0.13-0.36), respectively. The diagnostic odds ratio was 9.9 (95\% Cl: 5.1-19.3), but no heterogeneity was found for sensitivity and moderate heterogeneity was found for specificity (Figure 2). A hierarchical summary receiver operating characteristic curve for the diagnostic performance of endometrial volume for detecting endometrial malignancy is shown in Figure 3.

The Fagan nomogram shows that an increased endometrial volume increased the pretest probability of endometrial malignancy, from $8 \%$ to $16 \%$, whereas a normal finding decreased the pretest probability, from $8 \%$ to $2 \%$. No publication bias was observed $(p=0.43)$.

\section{DISCUSSION}

Most women with postmenopausal bleeding have a benign aetiology, and fewer than 8-10\% will be diagnosed with endometrial cancer. ${ }^{2,26}$ Two-dimensional ultrasound is the first step in the evaluation of women with postmenopausal bleeding with the measure of the endometrial thickness because it has been shown to be the most cost-effective strategy in this clinical setting. ${ }^{27,28}$ 


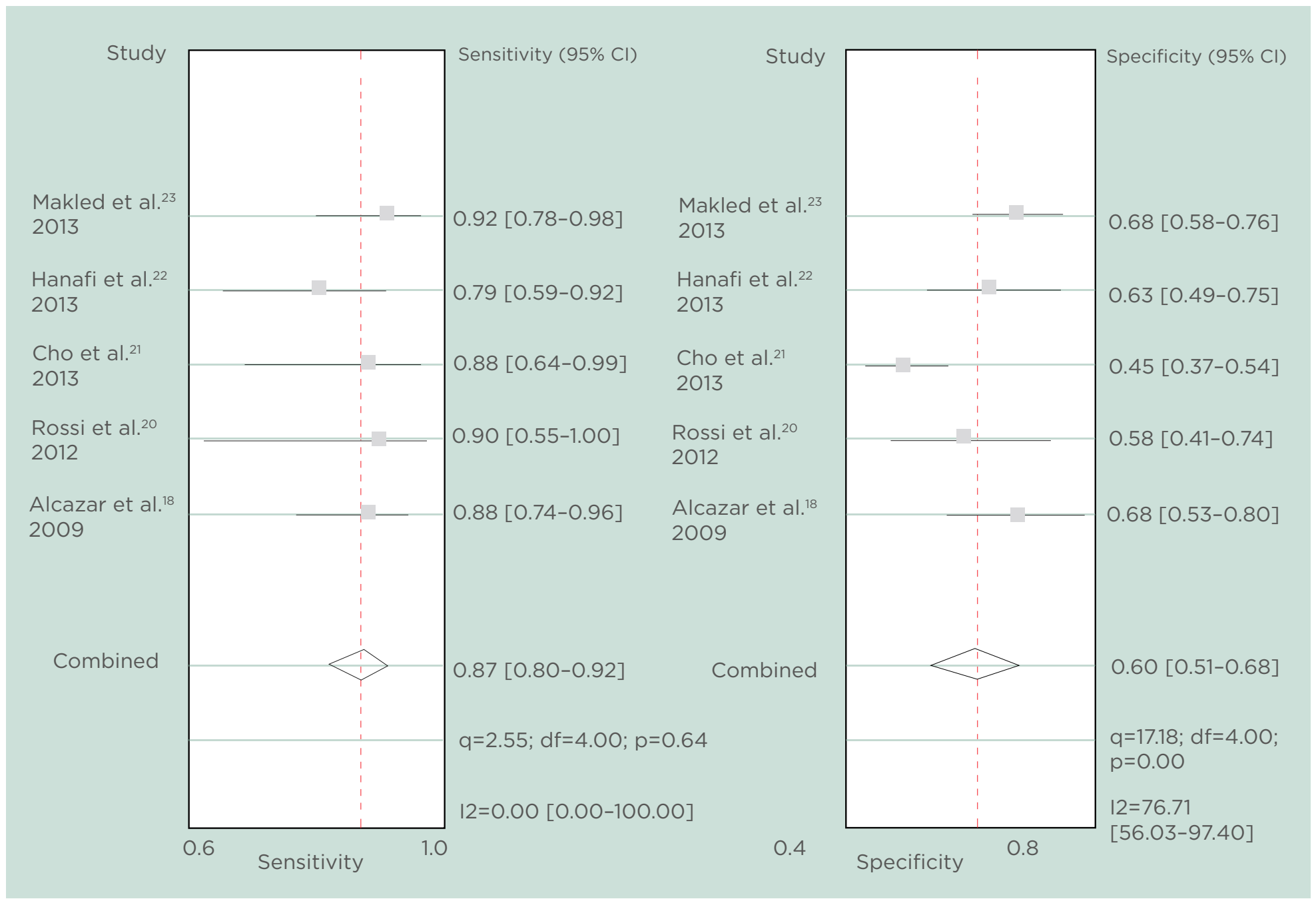

Figure 2: Forest plot for sensitivity and specificity for each study and pooled sensitivity and specificity for endometrial volume, after excluding studies contributing to heterogeneity.

Cl: confidence interval; df: degrees of freedom.

Several meta-analyses assessing the diagnostic performance of endometrial thickness for detecting endometrial cancer in women with postmenopausal bleeding,,29-32 and even in asymptomatic postmenopausal women, 33,34 have been reported. In women with postmenopausal bleeding, the most recent meta-analysis has demonstrated that an endometrial thickness $<5 \mathrm{~mm}$ is effective to rule out endometrial cancer, with a high sensitivity (96.2\%) and negative predictive value (99.3\%), but rather low specificity (51.5\%). ${ }^{2}$

The advent of 3D ultrasound allowed an accurate estimation of organ or structure volume..$^{35}$ There are different approaches for the estimation of organ volume, such as the use of the prolate ellipsoid measuring the three orthogonal diameters of the structure, using a distance and the perimeter of an ellipse, a spherical method, or the so-called VOCAL method. ${ }^{35-39}$ The latter processing system of the 3D volume allows calculation of the volume using a rotational method, with different rotation angles $\left(9^{\circ}, 15^{\circ}, 30^{\circ}\right)$.

The assessment of endometrial volume as measured by 3D ultrasound for detecting endometrial cancer in women with postmenopausal bleeding was first reported in $1996 . .^{10}$ In this study, Gruboeck et al. reported a series of 97 women with postmenopausal bleeding (11 had cancer). 


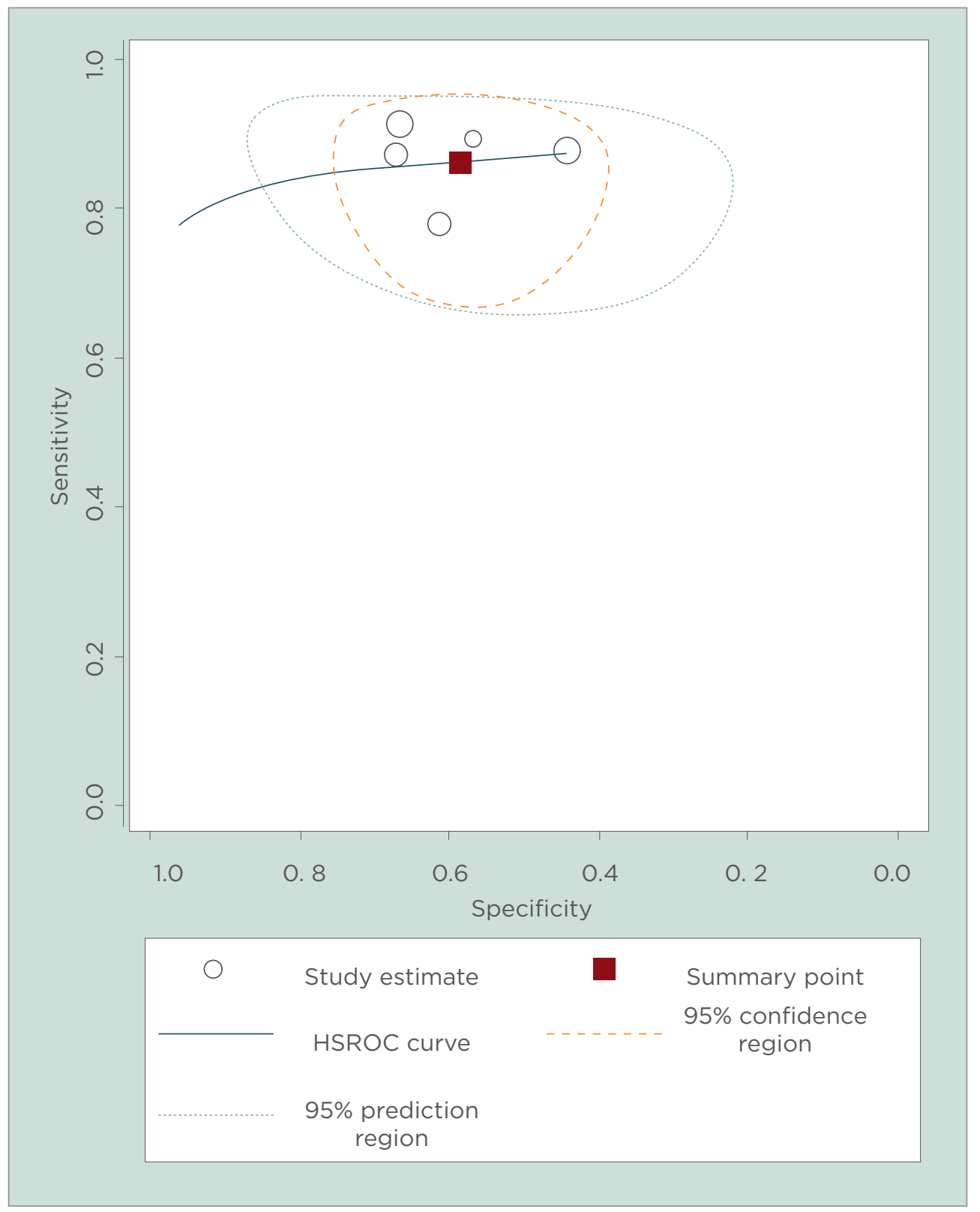

Figure 3: Hierarchical summary receiver operating characteristic curve for endometrial volume.

Area under the curve: 0.87 (95\% confidence interval: 0.84-0.90).

HSROC: hierarchical summary receiver operating characteristic.

They showed that an endometrial volume greater than $13 \mathrm{~mL}$ had a sensitivity and specificity of $100.0 \%$ and $98.8 \%$, respectively, for diagnosing endometrial cancer. ${ }^{10}$ However, no further study was reported in the subsequent 10 years. Since 2007, several studies have been published addressing this issue, all of them using the VOCAL method.

In the present meta-analysis, the authors have evaluated the diagnostic performance of endometrial volume as estimated by 3D ultrasound using the VOCAL method to predict the presence of endometrial malignancy in women with postmenopausal bleeding. In the meta-analysis, it was observed that pooled sensitivity and specificity of the endometrial volume were $87 \%$ (95\% Cl: $80-92 \%)$ and $60 \%$ (95\% Cl: 51-68\%), respectively, after excluding some papers that were identified as potential source of heterogeneity for a threshold effect.

The main strength of this study is that, to the best of the authors' knowledge, this is the first 
meta-analysis reported addressing this topic. Long et al. ${ }^{2}$ have reported a recent metaanalysis assessing the diagnostic performance of endometrial thickness for detecting endometrial cancer in women with postmenopausal women. ${ }^{2}$ In this meta-analysis, four studies were reported on, comprising data from 434 women, analysing 3D endometrial volume in this clinical setting. Out of these four studies, three have been included in this present meta-analysis $18,19,23$ and one was not because, from this paper, $2 \times 2$ tables could not be extracted. ${ }^{40}$ However, they did not perform an analysis about endometrial volume because of the small sample size.

However, the authors do consider there are some limitations that preclude drawing definitive conclusions regarding the role of endometrial volume as estimated by 3D ultrasound to detect endometrial cancer in women with postmenopausal bleeding.

First, the collected sample can be considered as relatively small as compared with that reported on meta-analyses focussed on endometrial thickness. The data presented here are based on 981 women derived from just eight studies, while meta analyses about endometrial thickness report data from 2,896 to 17,339 patients. ${ }^{29-32}$

On the other hand, as stated above, studies that used the VOCAL method for analysing the 3D volumes obtained during the exam, and estimating the endometrial volume, were selected because this method has been reported as the most accurate to estimate the volume of the endometrium. ${ }^{41}$ Raine-Fenning et al. ${ }^{24}$ described that employing a rotation step of less than $30^{\circ}$ was associated with a significantly smaller variance in measurements and a significantly greater mean endometrial volume. In this meta-analysis, most of the studies (in fact, all of them except those from Cho et al. ${ }^{21}$ and Alcázar et al. ${ }^{18}$ ) used a $30^{\circ}$ rotation step. This fact could be considered as a source of bias from the technical point of view, since the rotation angle used was not the most optimal for calculating the endometrial volume.

Furthermore, it is important to consider that in six studies the inclusion criteria were only patients with a thickened endometrium, >4 $\mathrm{mm}$, and this also may lead to a selection bias, leaving out from the analysis some cases of endometrial cancer present in symptomatic women with a thin endometrium. It should be borne in mind that $25-34 \%$ of the Type II endometrial cancer could be present in patients with thin or indistinct endometrium. ${ }^{42-43}$ The authors have no information about endometrial volume in these cases.

In addition, there was high heterogeneity among the studies relating to different cut-off values used for endometrial volume (1.35-5.3 $\mathrm{mL}$ ). The authors of the papers included were contacted in an attempt to perform a metaanalysis based on individual patient data, but none answered. Therefore, it is difficult to be precise about the specific cut-off value of endometrial volume to rule out endometrial cancer.

In most of the studies, endometrial hyperplasia with or without atypia cases were pooled in the benign group. There were no precise data for differentiating between the hyperplasia with and without atypia using the endometrial volume, so the authors had to be careful in the interpretation of that point, considering that almost $25 \%$ of patients with hyperplasia with atypia had a coexistent endometrial cancer in the final histology. ${ }^{44,45}$

Nevertheless, the authors could not compare the diagnostic performance of endometrial volume and endometrial thickness, of which is the current standard. ${ }^{46-48}$ Thus, it cannot be elucidated whether endometrial volume is better than endometrial thickness.

\section{CONCLUSION}

In conclusion, endometrial volume as estimated by 3D ultrasound using VOCAL software has a moderate diagnostic performance for detecting endometrial malignancy in women with postmenopausal bleeding. A rough comparison with the results from a recent meta-analysis focussed on endometrial thickness suggests that endometrial volume appears inferior to endometrial thickness. ${ }^{2}$ However, a formal metaanalytical comparison has not been performed so far. There is clear room for future research in this topic because better-designed prospective studies are needed. 


\section{References}

1. Ferlay $\mathrm{J}$ et al. Cancer incidence and mortality worldwide: sources, methods and major patterns in GLOBOCAN 2012. Int J Cancer. 2015;136:E359-86

2. Long $B$ et al. Ultrasound detection of endometrial cancer in women with postmenopausal bleeding: systematic review and meta-analysis. Gynecol Oncol. 2020;S0090-825830085-8.

3. Alcázar JL et al. Endometrial blood flow mapping using transvaginal power Doppler sonography in women with postmenopausal bleeding and thickened endometrium. Ultrasound Obstet Gynecol. 2003;21:583-8.

4. Ludwin A et al. Congenital Uterine Malformation by Experts (CUME): better criteria for distinguishing between normal/arcuate and septate uterus? Ultrasound Obstet Gynecol. 2018;51:101-9.

5. Alcázar JL. Three-dimensional ultrasound assessment of endometrial receptivity: a review. Reprod Biol Endocrinol. 2006;4:56.

6. Alcázar JL, Jurado M. Threedimensional ultrasound for assessing women with gynecological cancer: a systematic review. Gynecol Oncol. 2011;120:340-6.

7. Martins WP et al. Reliability and validity of tissue volume measurement by three-dimensional ultrasound: an experimental model. Ultrasound Obstet Gynecol. 2007;29:210-4

8. Alcázar JL et al. Endometrial volume and vascularity measurements by transvaginal 3-dimensional ultrasonography and power Doppler angiography in stimulated and tumoral endometria: an interobserver reproducibility study. J Ultrasound Med. 2005;24:1091-8.

9. Martins WP et al. A standardized measurement technique may improve the reliability of measurements of endometrial thickness and volume. Ultrasound Obstet Gynecol. 2011;38:107-15

10. Gruboeck K, Jurkovic D et al. The diagnostic value of endometrial thickness and volume measurements by three-dimensional ultrasound in patients with postmenopausal bleeding. Ultrasound Obstet Gynecol. 1996;8:272-6

11. Sotiriadis A et al. Synthesizing Evidence from Diagnostic Accuracy Tests: the SEDATE guideline. Ultrasound Obstet Gynecol 2016; 47:386-95

12. Whiting PF et al. QUADAS-2 a revised tool for the quality assessment of diagnostic accuracy studies. Ann Intern Med. 2011;155:52936.

13. European Network for Health
Technology Assessment (EUnetHTA). EUnetHTA guideline: meta-analysis of diagnostic test accuracy studies. 2014. https://eunethta.eu/wpcontent/uploads/2018/01/Metaanalysis-of-Diagnostic-Test-AccuracyStudies Guideline Final-Nov-2014. pdf. Last accessed: 21 April 2020.

14. Higgins JP et al. Measuring inconsistency in meta-analyses. BMJ. 2003;327:557-60.

15. Deeks JJ et al. The performance of tests of publication bias and other sample size effects in systematic reviews of diagnostic test accuracy was assessed. J Clin Epidemiol. 2005;58:882-93.

16. Mansour GM et al. Endometrial volume as predictor of malignancy in women with postmenopausal bleeding. Int J Gynaecol Obstet. 2007;99:206-10.

17. Yaman $\mathrm{C}$ et al. The role of threedimensional volume measurement in diagnosing endometrial cancer in patients with postmenopausal bleeding. Gynecol Oncol. 2008;110:390-5.

18. Alcazar JL, Galvan R. Threedimensional power Doppler ultrasound scanning for the prediction of endometrial cancer in women with postmenopausal bleeding and thickened endometrium. Am J Obstet Gynecol. 2009;200:44. e1-6.

19. Opolskiene $\mathrm{G}$ et al. Threedimensional ultrasound imaging for discrimination between benign and malignant endometrium in women with postmenopausal bleeding and sonographic endometrial thickness of at least $4.5 \mathrm{~mm}$. Ultrasound Obstet Gynecol. 2010;35:94-102.

20. Rossi A et al. Assessment of endometrial volume and vascularization using transvaginal 3D power Doppler angiography in women with postmenopausal bleeding. Int J Gynaecol Obstet. 2012;119:14-7.

21. Cho HJ et al. Investigations for postmenopausal uterine bleeding: special considerations for endometrial volume. Arch Iran Med. 2013;16:665-70

22. Hanafi S et al. Value of three dimensional power Doppler ultrasound in prediction of endometrial carcinoma in patients with postmenopausal bleeding. J Turk Ger Gynecol Assoc. 2014;15:78-81.

23. Makled AK et al. Three-dimensional power Doppler and endometrial volume as predictors of malignancy in patients with postmenopausal bleeding. J Obstet Gynaecol Res. 2013;39:1045-51.

24. Raine-Fenning $\mathbf{N}$ et al. The reproducibility of endometrial volume acquisition and measurement with the VOCAL-imaging program. Ultrasound Obstet Gynecol. 2002;19:69-75.

25. Mercé LT et al. Endometrial volume and vascularity measurements by transvaginal three-dimensional ultrasonography and power Doppler angiography in stimulated and tumoral endometria: intraobserver reproducibility. Gynecol Oncol. 2006;100:544-50.

26. Clarke MA et al. Association of endometrial cancer risk with postmenopausal bleeding in women a systematic review and meta-analysis. JAMA Intern Med. 2018;178:1210-22.

27. Clark TJ et al. Investigating postmenopausal bleeding for endometrial cancer: costeffectiveness of initial diagnostic strategies. BJOG. 2006;113:502-10.

28. Breijer MC et al. Diagnostic strategies for endometrial cancer in women with postmenopausal bleeding: cost-effectiveness of individualized strategies. Eur J Obstet Gynecol Reprod Biol. 2012;163:91-6.

29. Smith-Bindman $R$ et al. Endovaginal ultrasound to exclude endometrial cancer and other endometrial abnormalities. JAMA. 1998;280:1510-7.

30. Tabor A et al. Endometrial thickness as a test for endometrial cancer in women with postmenopausal vaginal bleeding. Obstet Gynecol. 2002;99:663-70.

31. Gupta JK et al. Ultrasonographic endometrial thickness for diagnosing endometrial pathology in women with postmenopausal bleeding: a meta-analysis. Acta Obstet Gynecol Scand. 2002;81:799-816.

32. Timmermans $A$ et al. Endometrial thickness measurement for detecting endometrial cancer in women with postmenopausal bleeding: a systematic review and meta-analysis. Obstet Gynecol. 2010;116:160-7.

33. Breijer MC et al. Capacity of endometrial thickness measurement to diagnose endometrial carcinoma in asymptomatic postmenopausal women: a systematic review and meta-analysis. Ultrasound Obstet Gynecol. 2012;40:621-9.

34. Alcázar JL et al. Risk of endometria cancer and endometrial hyperplasia with atypia in asymptomatic postmenopausal women with endometrial thickness $\geq 11 \mathrm{~mm}$ : a systematic review and meta-analysis. J Clin Ultrasound. 2018;46:565-70.

35. Alcázar JL. Three-dimensional ultrasound in gynecology: current status and future perspectives. Curr Womens Health Rev. 2005;1:1-14.

36. Pretorius DH et al. Three-dimensional ultrasound in obstetrics and 
gynecology. Radiol Clin North Am. 2001;39:499-521.

37. Jurkovic D. Three-dimensional ultrasound in gynecology: a critical evaluation. Ultrasound Obstet Gynecol. 2002;19:109-17.

38. Bega $\mathrm{G}$ et al. Three-dimensional ultrasonography in gynecology: technical aspects and clinical applications. J Ultrasound Med. 2003;22:1249-69.

39. Benacerraf BR. Three-dimensiona volume imaging in gynecology. Obstet Gynecol Clin North Am. 2019;46:755-81.

40. Kim A et al. Diagnostic utility of three-dimensional power Doppler ultrasound for postmenopausal bleeding. Taiwan J Obstet Gynecol. 2015;54:221-6.
41. Farrell $\mathrm{T}$ et al. The reliability and validity of three dimensional ultrasound volumetric measurements using an in vitro balloon and in vivo uterine model. BJOG. 2001;108:57382.

42. Wang $\mathrm{J}$ et al. Thin endometrial echo complex on ultrasound does not reliably exclude Type 2 endometrial cancers. Gynecol Oncol. 2006;101:120 5.

43. Billingsley $\mathrm{CC}$ et al. The use of transvaginal ultrasound in Type II endometrial cancer. Int J Gynecol Cancer. 2015;25:858-62.

44. Erdem B et al. Can concurrent high-risk endometrial carcinoma occur with atypical endometrial hyperplasia? Int J Surg. 2018;53:3503.
45. Travaglino A et al. Endometrial hyperplasia and the risk of coexistent cancer: WHO versus EIN criteria. Histopathology. 2019;74:676-87.

46. Munro MG; Southern California Permanente Medical Group's Abnormal Uterine Bleeding Working Group. Investigation of women with postmenopausal uterine bleeding: clinical practice recommendations. Perm J. 2014;18:55-70.

47. ACOG Committee opinion no 734: the role of transvaginal ultrasonography in evaluating the endometrium of women with postmenopausal bleeding. Obstet Gynecol. 2018;131:e124-9.

48. Evans D et al. No. 385-indications for pelvic examination. J Obstet Gynaecol Can. 2019;41:1221-34. 